\title{
Design of Cloud Robotic Services for Senior Citizens to Improve Independent living and Personal Health Management
}

\author{
M. Bonaccorsi ${ }^{*}$, L. Fiorini ${ }^{*}$, F.Cavallo, Member, IEEE, R. Esposito and P. Dario, \\ Member, IEEE
}

\begin{abstract}
A cloud robotics solution was designed and preliminary tested with a mobile robotic platform and a smart environment, in order to provide healthcare management services to senior citizens and improve their independent living. The solution was evaluate in terms of Quality of Service (QoS) and tested in the realistic scenario of the DomoCasa Living Lab, Peccioli, Italy. In particular, a medication reminding service, a remote home monitoring and an user indoor localization algorithm were outsourced in the cloud and provided to the robots, users and caregivers. The system pulled data from a smart environment and addressed the robot to the user for service delivery. Experiments demonstrated a service's Reliability of Respond at least of the $0.04 \%$ and a Time of Respond of the same order of magnitude of the processing time required by the user localization algorithm.
\end{abstract}

\section{Introduction}

EU population ageing is a long-term trend that will almost triple the share of those aged 80 years or above between 2011 and 2060. This will increase the demand for Nurse Practitioners (+94\% in 2025) [1] and Physician Assistants ( $+72 \%$ in 2025) [2] with several implications for quality of care and for the configuration of future costeffectiveness care delivery systems. One of the greater challenge in the next years will be to fit to the reduction of funds for social-medical services, with the opportunity to provide socially sustainable home care services to senior citizens. Seniors prefer to live as independently as possible [3] and to maintain their quality of life. Their wellbeing depends on the opportunity to efficiently managing medications and cares, and mainly lose their independence because of the difficulties in medication selfmanagement and health status monitoring [4]. Cognitive impairments and the com-

\footnotetext{
${ }^{*}$ Equal contribution to the work.
}

M. Bonaccorsi, L.Fiorini, F. Cavallo, R. Esposito and P. Dario are with The BioRobotics Institute, Scuola Superiore Sant'Anna, Viale Rinaldo Piaggio 34, 56025 Pontedera (PI), Italy (phone: +39 0587 672152; fax: +39050 883101; e-mail: m.bonaccorsi@sssup.it; 1.fiorini@sssup.it). 
plexity of the cares negatively impact on their independent living [5]. For these reasons, several works [6] investigated the opportunity to provide medication reminders, self-monitoring, and family therapy, and demonstrated that this could reduce hospitalizations from 33 to $69 \%$.

\section{Form Service Robotics to Cloud Service Robotics}

Service robotics for ageing population aim to support senior citizens with cognitive and physical impairments. In particular assistive robotic services were identified as a potential solution to improve quality of life enhancing mobility and communication, monitoring physiological parameters and daily activities [7]. Robots were developed to support elderly in the management of daily activities [8] and the monitoring of their health state $[9,10]$.

Many standalone and networked robots, also integrated in smart environments, was developed to perform specific tasks [11,12], acted as simple companion robot $[13,14]$ or provided complex assistive services $[15,16]$. Standalone robots autonomously planned and acted depending on their sensing capability and their internal model of the surrounding environment. These robots usually required high computational capabilities and expensive technologies that made them unaffordable [17]. Networked robots were defined as a group of autonomous mobile systems that made important use of wireless communications among them or with the environment and living systems, in order to fulfill complex tasks [18]. Smart environments, and intelligent agents, like wearable and personal devices, extended the effective sensing range of that robots and improved their planning and cooperation capability. Nevertheless, standalone and networked-robots faced inherent physical constraints as all computations were conducted onboard of the robots or on wireless devices, which had limited computational capabilities [19], and were proved to be insufficient to provide continuous and effective assistive services to fulfill seniors' needs. Recently cloud robotic paradigm extended the concept of networked robotics. Cloud robotics and cloud networked robotic paradigms were introduced by [20], to improve networked robotics and design a new generation of cheaper and smarter robots by exploiting cloud computing infrastructures. Main advantages in using cloud infrastructures, respect to classic client-servers, are (i) dynamically address physical and computational resources depending on the required workload for service delivery, (ii) improve quality of service by requiring no downtimes for hardware and software upgrades (iii) reduce costs because of no needs for hardware [21]. In cloud robotics, complex algorithms run on the cloud that acts as a modular and always connected remote brain. Cloud reduce time and energy expenditure for service delivery [22] and could improve the QoS [23] and the costeffectiveness of demanding AAL robotic services. 
Design of Cloud Robotic Services for Senior Citizens to Improve Independent living and Per- 3 sonal Health Management

Given the novelty of cloud infrastructures, several definitions of cloud robotics exists. In particular Ken Goldberg emphasized the benefits of the great computation capacity and memory allocation provided by the Cloud, to provide a new form of collective robot intelligence through learning and sharing paradigms [24,25].

In authors' opinion, Cloud Service Robotics (CSR) could be defined as the integration of different agents that allow an efficient, effective and robust cooperation between robots, smart environments and humans, to provide continuous services to senior citizens. CSR could be applied in many robotic applications, to offload CPU-heavy tasks and access base knowledge to expand robot consciousness beyond their physical body [19] (see Figure 1).

Two main paradigms were introduced in literature to improve service robotics: the Robot as a Service (RaaS) and the Software as a Service (SaaS) approaches. In the RaaS approach [23] the cloud is introduced to resolve issues on continuity of services. The relationship between users and robotic platforms is mediated by a robot management system on the cloud, that coordinates and selects the proper hardware platform to fulfill user needs and provide robotic services. In this model, the user is not required to own a specific robot, and the same robot could be used by several citizens.

The SaaS approach enables low cost robots, to offload storage and processing capabilities to the cloud. Complex algorithms on the cloud, could be provided on request, to improve reasoning, sensing and planning capability of robots with limited hardware. Recently, the SaaS paradigm was investigated to offload computational intensive tasks like object recognition and user localization. In the RobotEarth project [26], the knowledge required to recognize objects was opportunely aggregated and stored in a cloud infrastructure, and provided as a service to the connected robots in order to improve their interaction and manipulation capability.

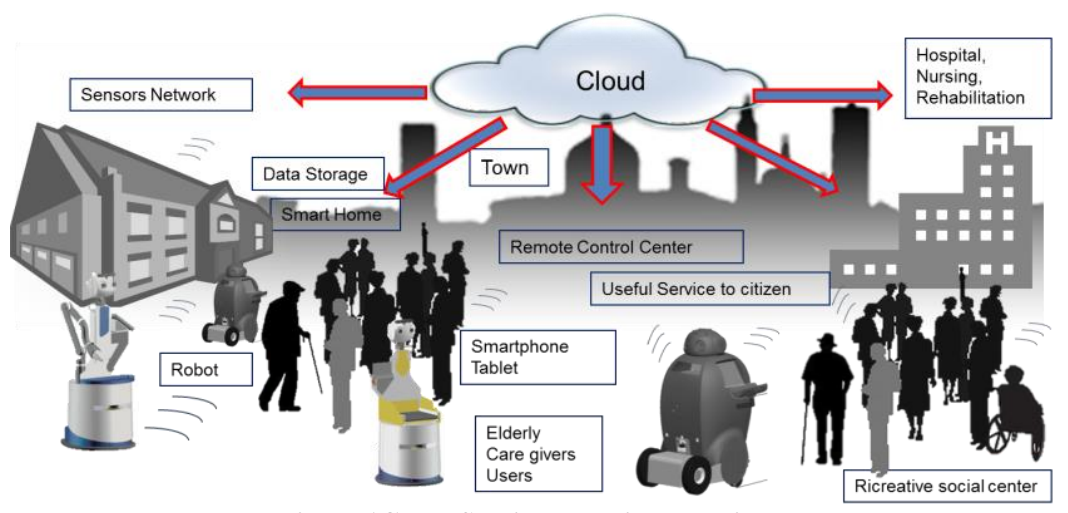

Figure 1Cloud Service Robotics paradigm 
Several researchers $[27,28]$ investigated the opportunity to provide Localization Based Services (LBS) by using cloud solutions, that are expected to improve the costeffectiveness and the QoS. User localization systems allow robots to know the position of the users without the need to implement a seeking procedure. in a cloud robotic solution, robots could inquire for user position on demand, and seniors' doesn't need to own computers running localization algorithms in their homes, reducing the system installation and maintenance costs.

\section{Proposed Services}

The proposed system was designed to provide high quality and continuous assistive services for independent living. According to RaaS paradigm, assistive robot was provided as a service, to alert the user in case of critical situations, and remind to take drugs or attend a medical therapy. Taking in account for SaaS paradigm, assistive software for user localization and environmental monitoring were developed, to improve robots functionalities (see Figure 2). In particular the system provided:

1. User indoor localization service - The system was able to locate the user to support robots in the continuous care service. A localization software pulled data from heterogeneous commercial and ad-hoc sensors, to estimate the position of the user. A sensor fusion approach was investigated to locate people in a robust and scalable manner. Accuracy and cost of the indoor localization service will depend on the typology and the number of the installed sensors. In case of a sensor fault, user position was be estimate, by fusing data from the remaining ones improving the reliability and the robustness of the service.

2. Care reminding service - A calendar for medication and care management was integrate in the system, and provided as a service to caregivers and users. This service was based onto Google Calendar tool. In this way, users and caregivers was allowed to schedule therapies and medical visits on the calendar. The system automatically addressed a robotic reminding service at the scheduled time, to remind the user about appointment or medication.

3. Environmental monitoring service - Data from sensors, like switches on doors, presence, light, humidity, temperature and GAS sensors, were collected and processed by a software module on the cloud. The software monitored the home status and alerted users and caregivers in case critical situations.

Low-cost companion robots could be improved by the proposed system, that allowed to perform environmental monitoring and user localization, independently to the robot 
Design of Cloud Robotic Services for Senior Citizens to Improve Independent living and Per- 5 sonal Health Management hardware.

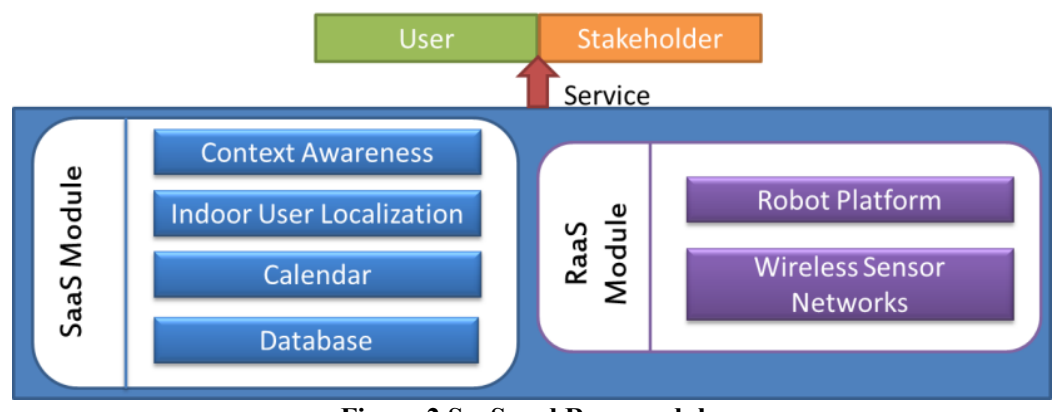

Figure 2 SaaS and Raas module

\section{System Architecture Description}

The proposed system was composed of four functional layers: (1) Hardware (2) Communication, (3) Application and (4) Interface layers, to provide assistive services to the users.

\subsection{Hardware Layer}

Hardware consisted of a mobile robotic platform and two ZigBee-based Wireless Sensor Networks (WSNs), one for user localization, and the other for environmental monitoring. The personal robot was based on a SCITOS G5 platform (Metralabs, Germany) that communicated with the user by means of an embedded touch screen. And exchanged data with the cloud by means of an Wi-Fi module. The user localization network was designed to locate multiple users at the same time, using the Received Signal Strength (RSS) [29]. It was composed of a ZigBee coordinator, a Data Logger (DL), a wearable mobile node and a set of ZigBee Anchors. The mobile node periodically sent messages to all anchors within one communication hop. Each anchor computed the RSS on the received messages and transmitted this value to the DL. The sensor network was developed for home monitoring and presence detection. It was composed of a ZigBee coordinator, a DL and a set of sensor nodes, that was connected to a selection of sensors, like Passive InfraRed (PIR) sensors, pressure sensor placed under a chair or a bed, switches on doors or drawers, temperature, humidity and light sensors. The two networks were set on different channels to avoid interferences and ensure the proper bandwidth for the localization and environmental monitoring services. Each DL was connected to a PC using USB connection, to upload data on the cloud platform. 


\subsection{Communication Layer}

Communication layer was composed of two tiers: Machine-to-Machine (M2M) and Machine-to-Cloud (M2C) communication modules. ZigBee, WI-FI and TCP/IP protocols were used for M2M communication to directly exchange data between the agents at the hardware layer. TCP/IP protocol connected the physical agents to the cloud in the $\mathrm{M} 2 \mathrm{C}$ communications, for data exchange and service delivery.

\subsection{Application Layer}

Application layer included a database (DB), a DB Management Software (DBMS), a User Localization Module (ULM), an Event Scheduler Module (ESM) and a Data Analysis Module (DAM). The DBMS managed DB entries and queries while the DB contained data from the connected robotic agents. The DB was composed of several tables: one for each observation from sensor outputs, one containing the list of installed sensors (typology and unique identification number) and another table recording the user estimated position. Outputs from physical agents and the estimated user position were sent to the DBMS and recorder in the DB. A dedicated localization algorithm was implemented by the ULM to estimate the user position. RSS observations were used to estimate user position by using Area-based localization [30] and trilateration [31] methods, while presence sensors were combined to improve positioning accuracy and perform host detection in a sensor fusion approach.

Numeric values $(\mathrm{x}, \mathrm{y})$ and semantic information on user position were provided to the robots to efficiently provide assistive localization based services. The ESM knew the current date time, user's commitments and appointments and scheduled the reminding services. After scheduling, at the proper time it retrieved the user position stored in the DB and tells the robot to reach the user and provide required reminding service. The DAM, periodically analyzed the data in the DB and alerted the system in case of critical situations.

\subsection{Interface layer}

The interface layer consisted of a Web application for remote home monitoring. It was connected directly to the DB on the same remote server with a public static IP,

Figure 3 Web interface for home monitoring 
Design of Cloud Robotic Services for Senior Citizens to Improve Independent living and Per-

sonal Health Management

and the access was restricted to authorized people only. The interface home page provided mean light, humidity, and temperature values for each sensitized room (see Figure 3) and the entrance door status. An alarm webpage provided a list of alarms that occurred, while the localization webpage reported the room where the user was located.

\section{Application Scenarios}

The systems was preliminary evaluated and tested in the realistic scenario of the DomoCasa Living Lab, Peccioli, Italy; a $200 \mathrm{~m}^{2}$ flat with a living room, a kitchen, a restroom and two bedrooms. DomoCasa was instrumented with the previously described service robot and the two WSNs. In particular, fifteen anchors, six PIRs, and five sensorized carpets and pillows were installed for user localization. A PC called domocasa-PC gathered all the sensor outputs and sent them to another PC, named remote-PC, that acted as a cloud and implemented the assistive robotic services. In the application scenario, the user stood wherever he wanted in the kitchen and the companion robot, at the scheduled times, automatically moved from the living-room to reached him/her and remind to take drugs. After the interaction with robot, the user gave a feedback for the reminding service through a specific app installed in the robot's tablet. Data from the SE were sent to the cloud for home monitoring and critical situation recognition. The web interface on the robot's tablet allowed to check the status of the house and the calendar (see Figure 4 and Figure 5).

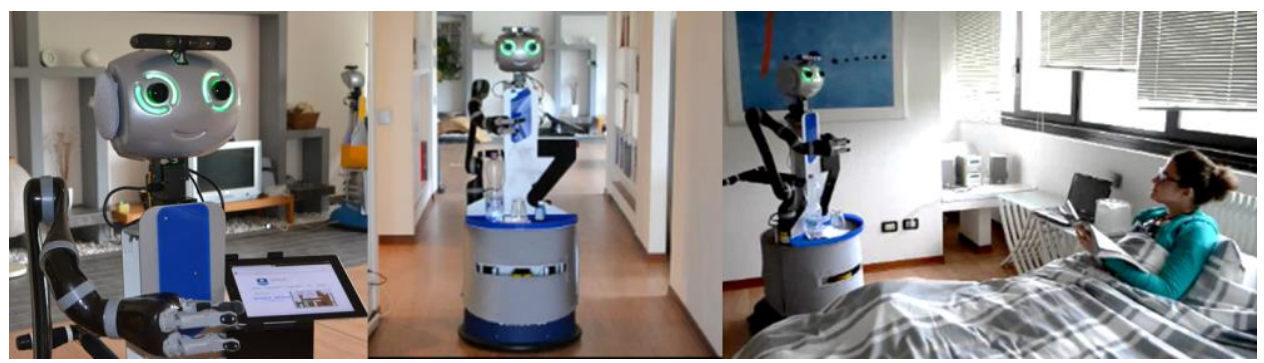

Figure 4 Storyboard of the proposed service. The domestic robot reached the user in the bedroom and acted as a physical reminding

\section{Evaluation Metrics}

The performance of the cloud platform was estimated through two parameters: the Time of Respond (ToR) and the Reliability of Respond (RoR) [32]. The ToR was defined as "the time needed from a client to receive a response after a request of 
service". The RoR instead was defined as "the measure of confidence that the retrieval data is free from errors" and its value was given in percent and calculated as the ratio between the succeeded requests and the total request. The RoR was computed as the ratio between DB data recovery which has been successful and the total requests.

The user localization service was designed and preliminary evaluated according to state of the art metrics regarding system usability and cost [33,34], while the accuracy will be evaluated in future experimentations.

\section{Results}

Quality of service was assessed by the analysis of the system ToR and RoR in the DomoCasa environment. System total time of service was $65.56 \mathrm{~ms}$ plus transmission time. Furthermore, in Table 1 were reported the complete results of ToR analysis. ToR was divided into two value: (i) ToR to save data into the DB (ToR_DB), which was obtained by mediating the ToT DB provided by each sensor typology, and (ii) ToR related to service requests (ToR_SR) was the time that system needs to provide the user position to the client which made the request. RoR value was computed as the ratio between successfully requests from the ESM for user position and its total request to the DB. This module inquired for user position at the rate of $1 \mathrm{~Hz}$. The number of service fails was less than $0.04 \%$.

Table 1. ToR values in milliseconds.

\begin{tabular}{ll}
\hline Parameter & Time \\
\hline Mean ToR_DB over all data & $32.08[\mathrm{~ms}]$ \\
Mean ToR_SR & $24.27[\mathrm{~ms}]$ \\
Mean time of processing for Localization & $9.21[\mathrm{~ms}]$ \\
\hline
\end{tabular}

The overall cost of the communication between the sensor networks and the user localization module on the cloud, was comparable with internet cost. Interference between the wireless sensors in DomoCasa were avoided by setting the localization and the sensor networks on different ZigBee channels. The sensor network nodes were provided by the plug-and-play feature, that allow them to automatically join the network and send data at the start-up, in order to reduce the installation complexity. The localization system was able to locate multiple users at the same time, thanks to the synergic use of information coming from the ZigBee wearable radio devices and presence sensos in the environment. Thanks to the cloud DB, the user's movement could be recorded for history behavioral models and analysis for up to 1 year. 


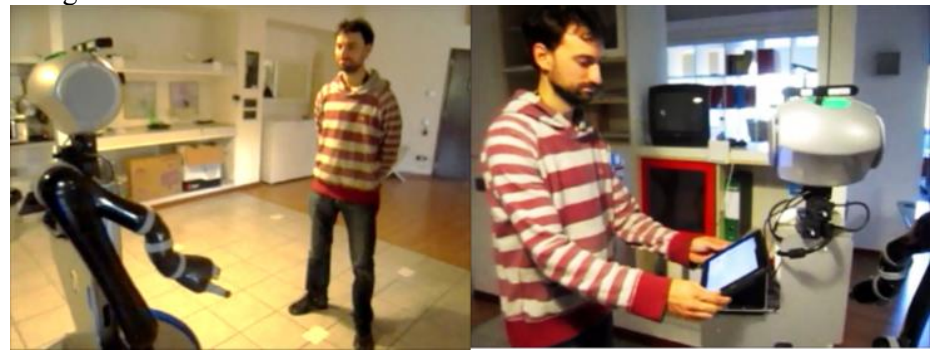

Figure 5 Storyboard of the proposed service. The domestic robot reached the user in the kitchen and acted as a physical reminding

\section{Acknowledgment}

The research leading to these results has received funding from the European Community's Seventh Framework Program (FP7/2007-2013) under grant agreement no. 288899 (Robot-Era Project). This work was also supported in part by Telecom Italia, Joint Open Lab WHITE, Pisa, Italy.

\section{References}

1. Auerbach, D. I. "Will the NP workforce grow in the future?: New forecasts and implications for healthcare delivery". Medical care, 2012, 50.7: 606-610.

2. Hooker, R. S., Cawley, J. F., and Christine M. Everett. "Predictive modeling the physician assistant supply: 2010-2025." Public Health Reports 126.5 (2011): 708.

3. Stula, S. : "Living in Old Age in Europe - Current Developments and Challenges". Working Paper No. 7 of the Observatory for Sociopolitical Developments in Europe. 2012. Available at: http://www.sociopoliticalobservatory.eu/uploads/tx_aebgppublications/AP_7_EN.pdf

4. Lieto, J. M., and Schmidt, K. S. : "Reduced ability to self-administer medication is associated with assisted living placement in a continuing care retirement community." Journal of the American Medical Directors Association 6.4 (2005): 246-249.

5. Kokurek, B. "Promoting medication adherence in older adults... and the rest of us". Diabetes Spectrum, 2009, 22.2: 80-84.

6. Ostemberg, L. et al. "Adherence to medication. New England Journal of Medicine", 2005, 353.5: 487-497.

7. Tiwari, P., et al. :"Some Non-technology Implications for Wider Application of Robots to Assist Older People." Health Care and Informatics Review Online (2010).

8. "Catalogue of Projects", Ambient Assisted Living Joint Programme, 2013. Resource available at: http://www.aal-europe.eu/wp-content/uploads/2013/09/AALCatalogue2013_Final.pdf

9. DOMEO project. website: www.aal-domeo.eu, 2012.

10. Lowet, D., "Florence - A Multipurpose Robotic Platform to Support Elderly at Home", Proc of the 2012 Workshop on Ambient Intelligence Infrastructures (WAmIi), Nov 2012.

11. Iwata, H., Sugano, S. :"Design of human Symbiotic Robot Twendy-one”. IEEE Interational Conference on Robotics and Automation. 2009. Pages: 580-586

12. K. Wada and T. Shibata. :"Robot therapy in a care house: Its sociopsychological and physiological effects on the residents," in Proc. 2006 Int. Conf. Robotics and Automation, Orlando, FL, 
May 2006, pp. 3966-3971.

13. W. D. Stiehl, J. Lieberman, C. Breazeal, L. Basel, and L. Lalla, "The design of the huggable: A therapeutic robotic companion for relational affective touch," presented at AAAI Fall Symp. Caring Machines: AI in Eldercare, Washington, DC, 2005

14. M. R. Banks, L. M. Willoughby, and W. A. Banks, "Animal-assisted therapy and loneliness in nursing homes: Use of robotic versus living dogs," J. Amer. Med. Dir. Assoc., vol. 9, no. 3, pp. 173-177, 2008

15. A. Badii, et al., "CompanionAble: Graceful integration of mobile robot companion with a smart home environment". Gerontechnology, 8(3), 181, 2009.

16. F. Cavallo et al. "Improving Domiciliary Robotic Services by Integrating the ASTRO Robot in an AmI Infrastructure." Gearing Up and Accelerating Cross-fertilization between Academic and Industrial Robotics Research in Europe:. Springer International Publishing, 2014. 267-282.L

17. E. Guizzo, "Robots with their heads in the clouds." Spectrum, IEEE 48.3 (2011): 16-18.

18. A. Sanfeliu, N. Hagita, A. Saffiotti, "Special issue: Network robot systems. Robotics and Autonomous Systems", 56(10), 791-791, 2008

19. G. Hu, W. P. Tay, and Y. Wen, "Cloud Robotics: Architecture", Challenges and Applications, IEEE Network, 2012, pp.21-28.

20. Kamei, Koji, et al. "Cloud networked robotics." Network, IEEE 26.3 (2012): 28-34.M

21. Abidi, F., et al. "Cloud servers vs. dedicated servers-A survey." Innovation and Technology in Education (MITE), 2013 IEEE International Conference in MOOC. IEEE, 2013.

22. Kumar, K., et al. "Cloud computing for mobile users: Can offloading computation save energy?." Computer 43.4 (2010): 51-56.

23. Z.Du et al., "Design of a Robot Cloud Center", Proc. IEEE IDAS 2011, 2011, pp. 269-275.

24. K. Goldberg and B. Kehoe, Cloud Robotics and Automation: A Survey of Related Work. UC Berkeley Technical Report No. UCB/EECS-2013-5. Available: http://www.eecs.berkeley.edu/Pubs/TechRpts/ 2013/EECS-2013-5.html, 2013.

25. The RoboEarth project. Official website available at: http://roboearth.org/, 2014.

26. M.Waibel, et al., RoboEarth, IEEE Robotics and Automation Magazine, 2011, pp 69-82.

27. Y. Wang, et al., "Integration of Range-Based and Range-Free Localization Algorithms in Wireless Sensor Networks for Mobile Clouds", Green Computing and Communications (GreenCom), 2013 IEEE and Internet of Things (iThings/CPSCom), IEEE International Conference on and IEEE Cyber, Physical and Social Computing. IEEE, 2013.

28. X. Zhu, C. Qiu , Y. Tao and Q. Jin, Cloud based localization for mobile robots, Workshop at IROS 2013 on cloud computing.

29. T.A. Alhmiedat and S.H. Yang. :"A ZigBee based mobile tracking system through wireless sensor network". International Journal of Advanced Mechatronic Systems. Vol. 1. N¹. 2008.

30. J. Blumenthal, et al., Localization in ZigBee based sensor networks"; 1st European ZigBee Developer's Conference (EuZDC), München-Dornach, Deutschland, 2007

31. Y. Wang, et al., :"Bluetooth positioning using RSSI and triangulation methods". Consumer Communications and Networking Conference (CCNC). 2013.

32. L.Wang, M.Liu, M. Q.H Meng, R.Siegwart, Towards Real-Time Multi-Sensor Information Retrieval in Cloud Robotic System, IEEE International Conference on MFI, September 13-15, 2012, Hamburg, Germany.

33. S.Eisa, A.Moreira, "Requirements and Metrics for Location and Traking for Ambient Assisted Living”, Int. Conf. on Indoor Positioning and Indoor Navigation, 13-15 November 2012.

34. C. Fabbricatore, M.Zucker, S. Ziganki, A.P. Karduck, "Toward an Unified Architecture for Smart Home and Ambient Assisted Living Solutions”, IEEE DEST, 2011. 